\section{Hot Topic}

\section{Triple-Therapie mit einem Inhalator}

Vestbo J et al. Single inhaler extrafine triple therapy versus long-acting muscarinic antagonist therapy for chronic obstructive pulmonary disease (TRINITY): a double-blind, parallel group, randomised controlled trial. Lancet 2017; DOI: 10.1016/S0140-6736(17)30188-5

Patienten mit COPD und hohem Exazerbationsrisiko erhalten inhalativ lang-wirksame Muskarinantagonisten, Kortikosteroide plus B2-Sympathomimetika oder die Dreifachkombination. Die TRINITY-Studie überprüfte die Effekte von Tiotropium und TripleTherapie auf die Exazerbationsrate sowie die Nichtunterlegenheit einer Fixkombination in 1 Inhalator. Dabei soll die extrafeine Formulierung im Pulverdevice die Wirkung auf die kleinen Atemwege verbessern.

TRINITY war eine doppelblinde Parallelgruppenstudie, an der 15 Länder teilnahmen. Einschlusskriterien für die insgesamt 2691 Patienten waren ein Lebensalter $>40$ Jahre, eine $\mathrm{FEV}_{1}<50 \%, \mathrm{FEV}_{1}$ | FVC $<70 \%$ und $\geq 1$ Exazerbation im Jahr vor der Randomisierung. Die Behandlung erfolgte über 52 Wochen in 3 Gruppen:

- Tiotropium ( $n=1076)$,

- Beclometason/Formoterol/Glykopyrronium ( $n=1077 ; 1$ Inhalator, Fix-Triple),

- Beclometason/Formoterol und Tiotropium(n= 537; 2 Inhalatoren, Open-Triple).

Die Compliance lag in allen Behandlungsgruppen über $94 \%$. Die durchschnittliche Behandlungsdauer betrug 365 Tage. Im Tiotropium-Arm kamen Therapieabbrüche häufiger vor ( $15 \%$ vs. $9 \%$ vs. $8 \%$ ). Mittelschwere und schwere Exazerbationen waren in beiden Triple-Gruppen seltener als bei der Monotherapie. Verglichen mit Tiotropium reduzierte Fix-Triple die Exazerbationsrate um 20\% (Relatives Risiko RR 0,80; 95\%-Konfidenzintervall KI 0,69 $-0,92 ; p=0,0025)$. Zwischen den Kombinationsarmen bestand kein signifikanter Unterschied. Fix- und Open-Triple verlängerten darüber hinaus die Zeit bis zur ersten Exazerbation (Hazard Ratio 0,70;
$95 \%-K I 0,52-0,95 ; p=0,0208)$. In Woche 52 betrug die Prä-Dosis-FEV1 0,082 L (FixTriple), 0,085 L (Open-Triple) und 0,021 L (Tiotropium). Die Kombinationstherapien waren Tiotropium während der gesamten Behandlungsperiode zu allen Messzeitpunkten überlegen. Die Ansprechraten (Verbesserung um $\geq 100 \mathrm{ml}$ ) von Fix- und Open-Triple in Woche 26 und 52 waren vergleichbar, aber höher als die von Tiotropium. Dies schlug sich im SGRQ (Saint Georg Respiratory Questionnaire) mit deutlicheren Verbesserungen nieder. In der Tiotropium-Gruppe benötigten die Patienten häufiger die Rescue-Medikation Salbutamol. Dies galt für die Anzahl der Hübe und der Verwendungstage.

Komplikationen waren insgesamt selten und überwiegend leicht bis mittelschwer. An einer Pneumonie erkrankten $1 \%$ (Tiotropium), 2\% (Fix-Triple) und 2\% (Open-Triple). In jeder Gruppe traten bei $1 \%$ ischämisch induzierte Herzprobleme auf. Therapieassoziierte Nebenwirkungen lagen zwischen $<1 \%$ und $2 \%$. Dabei handelte es sich um Mundtrockenheit, Muskelkrämpfe, Heiserkeit und orale Candidiasitiden. In der Tiotropium-Gruppe wurde 1 Angina pectoris auf die Medikation zurückgeführt.

FAZIT

Die extrafeine Fix-Triple-Therapie war Tiotropium überlegen und der OpenTriple-Anwendung nicht unterlegen. Besonders profitierten Patienten mit häufigen Exazerbationen und die Subgruppe mit Eosinophilie, in der das Exazerbationsrisiko um $30 \%$ geringer war. Die Eosinophilie sagte die Ansprechwahrscheinlichkeit voraus. Die Autoren stellten die verstärkte Effektivität der Kombinationsbehandlungen bereits bei einem Wert von $2 \%$ Eosinophilen im peripheren Blut fest.

Dr. med. Susanne Krome, Melle

\section{Kommentar}

Die TRINITY-Studie läutet das Zeitalter der inhalativen Fix-Triple-Therapie in der Behandlung der COPD ein. Auf den ersten Blick scheinen die Ergebnisse durchaus trivial, bei genauerer Betrachtung werfen sie jedoch Fragen für die Wissenschaft und den klinischen Alltag auf. Unter Berücksichtigung anderer rezenter Studienergebnisse (z.B. FLAME-Studie) ist zunächst darauf zu verweisen, dass die Überlegenheit einer LAMA-LABAICS-Kombination gegenüber einer reinen LAMA-LABA-Kombination durch das vorliegende Studienkonzept nicht beantwortet wurde. Die Überlegenheit in der Reduktion von Exazerbationen durch die Triple-Therapie gegenüber einer LAMAMonotherapie könnte „lediglich“ ein Effekt der verstärkten Bronchodilatation sein, ein additiver Effekt einer ICS-Therapie ist weder ein- noch auszuschließen. Dies ist insofern von Bedeutung, da GOLD 2017 im Vergleich zu den vorangegangenen Jahren wesentlich restriktiver in der Nutzung gegenüber ICS auftritt. Darüber hinaus ist darauf zu verweisen, dass TRINITY eine zweimal tägliche Inhalationstherapie mit Glykopyrronium als Teil einer Fixkombination (LAMA/LABA und LAMA/LABA/ICS) in einem Inhalator verwendete. Die Grundlage dieses Behandlungskonzeptes ist konsistent mit der pharmakologischen Halbwertszeit von Glykopyrronium. Dies steht jedoch in einem scheinbaren Widerspruch zur derzeit geläufigen Verwendung der Fixkombination von Glykopyrronium mit Indacaterol in einmal täglicher Applikationsform.

Aus rein klinischer Sicht könnte die zukünftige inhalative Fix-Triple-Kombination das Therapieregime vereinfachen, die Anzahl an erforderlichen Inhalatoren auf eine Basistherapie und eine Bedarfsmedikation reduzieren und damit potentiell die Therapietreue der Patienten erhöhen. Die Gefahr, die sich jedoch hinter einer inhalativen „Maximaltherapie“ verbirgt, ist eine unkritische Verordnung, ungeachtet vom klinischen Phänotyp. Obwohl die Autoren der Arbeit sehr gezielt nur Patienten mit mittel-schwergradiger COPD und Exazerbationsanamnese eingeschlossen haben, zeigen Daten aus 
der klinischen Praxis, dass bereits heute die überwiegende Mehrzahl dieser Patienten eine inhalative Triple-Therapie in unterschiedlichen Kombinationsvarianten erhalten (LAMA/LABA+Mono-ICS, LABA/ICS + Mono-LAMA). Die Verfügbarkeit einer fixen Triple-Therapie könnte dieses Problem noch weiter verstärken bzw. zu noch mehr Ratlosigkeit und Polypharmazie in der Primärversorgung von betroffenen Patienten führen. Somit warten wir nicht nur auf weitere Forschungsergebnisse, um die so oft geforderte individualisierte COPD-Therapie zu untermauern, sondern auch auf praxisrelevante Empfehlungen wissenschaftlicher Gesellschaften und/oder Gesundheitsbehörden, die verschreibenden Ärztinnen und Ärzten tatsächlich einen Pfad aufzeichnen wie, wann, wie oft und welche Inhalationstherapie denn jetzt bei COPD zu Beginn der Erkrankung und bei Progress empfohlen werden kann.

PD Dr. Arschang Valipour, Wien 